\title{
Viral hemorrhagic fever
}

INSERM

\section{Source}

INSERM. (1999). Orphanet: an online rare disease and orphan drug data base. Viral hemorrhagic fever. ORPHA:341

Viral hemorrhagic fever is a group of recently discovered contagious viral infections characterized by severe, multiple, and often fatal hemorrhages. African fevers include Lassa fever discovered in 1969, Marburg's disease that first occurred in 1967, and Ebola fever that appeared in 1976. Other viruses may also cause hemorrhagic fevers (for example, arbovirus fever). 